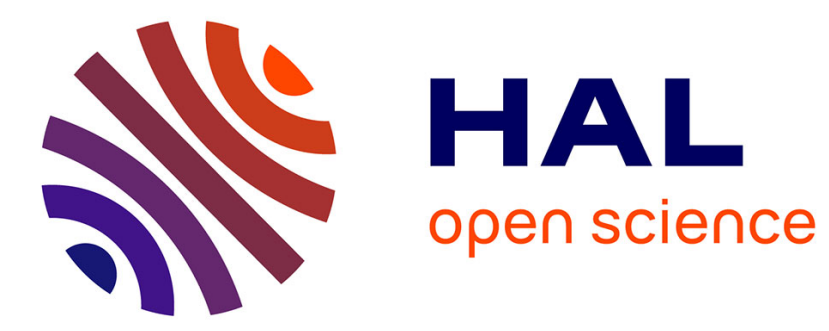

\title{
Pollution, décès prématuré et compensation
}

Grégory Ponthière

\section{To cite this version:}

Grégory Ponthière. Pollution, décès prématuré et compensation. 2014. hal-01095459

\section{HAL Id: hal-01095459 \\ https://hal-pjse.archives-ouvertes.fr/hal-01095459}

Preprint submitted on 15 Dec 2014

HAL is a multi-disciplinary open access archive for the deposit and dissemination of scientific research documents, whether they are published or not. The documents may come from teaching and research institutions in France or abroad, or from public or private research centers.
L'archive ouverte pluridisciplinaire HAL, est destinée au dépôt et à la diffusion de documents scientifiques de niveau recherche, publiés ou non, émanant des établissements d'enseignement et de recherche français ou étrangers, des laboratoires publics ou privés. 


\section{PARISSCHOQL OFECQNOMICS}

WORKING PAPER N 2014 - 40

Pollution, décès prématuré et compensation

Grégory Ponthière

JEL Codes: I31, J10, Q50

Keywords: Pollution, Mortalité, Mortalité prématurée, Compensation.

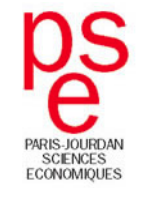




\title{
Pollution, décès prématuré et compensation*
}

\author{
Gregory Ponthiere ${ }^{\dagger \ddagger}$
}

December 5, 2014

\begin{abstract}
Nous étudions la compensation des personnes décédées prématurément dans une économie où la production génère de la pollution, et où la pollution réduit, au-delà d'un certain seuil, les chances de survie. Pour ce faire, nous caractérisons l'optimum égalitarien ex post et nous le comparons à l'équilibre de laissez-faire et à l'optimum utilitariste. Lorsque le seuil de pollution au-dessus duquel une mortalité prématurée apparait est élevé, l'optimum égalitarien ex post requiert une pollution égale à ce seuil, et inférieure à celles prévalant au laissez-faire et à l'optimum utilitariste. Mais lorsque le seuil critique de pollution est faible, le niveau de pollution associé à l'optimum égalitarien ex post est égal à celui qui prévaut au laissez-faire, et supérieur à celui associé à l'optimum utilitariste.

Mots clés: pollution, mortalité, mortalité prématurée, compensation.

JEL keywords: I31, J10, Q50.
\end{abstract}

\footnotetext{
*L'auteur remercie Arnold Chassagnon et Fabrice Etilé pour leurs commentaires et suggestions sur ce travail.

${ }^{\dagger}$ Université Paris Est (ERUDITE) et Paris School of Economics. Adresse: ENS, 48 boulevard Jourdan, bâtiment A, bureau 201, 75014 Paris. E-mail: gregory.ponthiere@ens.fr

‡L'auteur bénéficie du soutien de l'ANR EQUIRISK (Equity in Risky Economic Environments) (ANR-12-INEG-0006-01)
} 


\section{Introduction}

La pollution de l'environnement - pollution de l'air, des sols, de l'eau - constitue un facteur de mortalité de premier plan. ${ }^{1}$ Dans une étude longitudinale portant sur 6 villes américaines durant la période 1974-1998, Laden et al (2006) ont estimé qu'une hausse de la concentration de particules fines $\mathrm{PM}_{2.5}$ de 10 $\mu \mathrm{g} / \mathrm{m}^{3}$ cause une surmortalité de $15 \%{ }^{2}$ Dans une récente étude, l'Organisation Mondiale de la Santé (2014) estime qu'en 2012, environ 7 millions de personnes sont décédées à cause d'une pollution de l'air. ${ }^{3}$

La surmortalité causée par la pollution de l'environnement est à l'origine d'inégalités dans les durées de vie. Etant donné que la longévité constitue une dimension essentielle du bien-être humain - nécessaire afin d'avoir une "vie bonne", quelle que soit la conception de la "vie bonne" à laquelle on adhère mourir prématurément constitue une cause de déprivation majeure (Sen 1998). ${ }^{4}$ Les inégalités de longévité liées à la pollution génèrent donc des fortes inégalités de bien-être entre les hommes.

Les victimes de la pollution ne peuvent pas être considérées comme responsables de leur sort, et ce pour au moins deux raisons. D'une part, un individu pris isolément a une influence infime sur le processus de pollution; celui-ci se situe donc largement en dehors de son contrôle. D'autre part, la mortalité liée à la pollution frappe les individus d'une manière arbitraire. Par conséquent, si on applique le principe de compensation énoncé par Fleurbaey (2008), les pouvoirs publics devraient chercher à réduire les écarts de bien-être associés à cette surmortalité, car celle-ci résulte, au niveau individuel, de circonstances sur lesquelles l'individu ne peut pas agir.

L'objectif de cette étude est d'analyser la compensation des victimes de la pollution. Comme cela a été discuté antérieurement (Fleurbaey et Ponthiere 2013, Fleurbaey et al 2014a), la compensation des personnes disparues prématurément semble, à première vue, impossible. Ex ante, il est difficile d'identifier les personnes qui connaitront un décès prématuré, tandis qu'ex post (une fois la durée de vie révélée), il est trop tard pour les compenser. Malgré ces difficultés, ces travaux ont montré qu'il était possible de minimiser les écarts de bien-être entre les individus à vie courte et à vie longue. A cette fin, l'optimum égalitarien ex post implique des profils de consommation décroissants avec l'âge, voire un report de l'âge de départ à la retraite (Fleurbaey et al 2014b).

La présente étude vise à compléter ces travaux en considérant un environnement où la pollution, issue de l'activité de production, réduit la durée de vie de certaines personnes. Pour ce faire, nous allons d'abord caractériser le laissezfaire et l'optimum utilitariste, puis comparer ceux-ci à l'optimum égalitarien $e x$

\footnotetext{
${ }^{1}$ Sur les dimensions environnementales affectant la mortalité, voir Sartor (2002).

${ }^{2}$ Voir également Finkelstein et al (2003).

${ }^{3}$ La plupart de ces décès se situent en Asie. 4.3 millions de décès sont liés à la pollution intérieure (indoor air pollution), et 3.7 millions de décès sont liés à la pollution extérieure (outdoor air pollution). Un million de décès sont causés par une double exposition.

${ }^{4}$ Ce fait est confirmé par les nombreuses études quantifiant les gains ou pertes de bien-être associés à des variations de longévité. Voir notamment Usher $(1973,1980)$, et, plus récemment, Costa et Steckel (1997), Becker et al (2005) et Fleurbaey et Gaulier (2009).
} 
post, définie comme l'allocation maximisant le bien-être réalisé sur la vie de la personne la moins bien lottie dans la population.

Anticipant nos résultats, nous montrons que lorsque le seuil de pollution audessus duquel une mortalité prématurée apparait est élevé, l'optimum égalitarien ex post requiert une pollution égale à ce seuil, et donc inférieure à celle du laissezfaire et de l'optimum utilitariste. Par contre, lorsque ce seuil critique est faible, le niveau de pollution associé à l'optimum égalitarien ex post est égal à celui prévalent au laissez-faire, et supérieur à celui associé à l'optimum utilitariste.

Le reste de cette étude est organisée comme suit. La section 2 présente le modèle. L'équilibre du laissez-faire est caractérisé dans la section 3. L'optimum utilitariste est étudié dans la section 4, puis est comparé à l'optimum égalitarien ex post dans la section 5 . La section 6 conclut.

\section{Le modèle}

Nous étudions une économie dont la population est un continuum de taille unitaire. Les individus vivent soit deux périodes, soit une seule période. La durée de chaque période est normalisée à 1 . Chaque individu travaille une fraction $\ell$ de la première période, et, en cas de survie, est retraité en seconde période. L'activité de production génère de la pollution. Cette pollution, au delà d'un certain seuil, réduit les chances de survie.

Nous supposons que la fonction de production prend la forme suivante:

$$
Y=A \ell
$$

où $Y$ est l'output, $A$ est un paramètre de productivité, et $\ell$ est la quantité de travail $(0 \leq \ell \leq 1)$.

La pollution $P$ est proportionnelle à l'output:

$$
P=\eta Y=\eta A \ell
$$

$\eta>0$ reflète le caractère polluant du processus de production. Une interprétation simple de cette relation est de considérer qu'une production $Y$ requiert une production d'énergie qui génère des émissions polluantes égales à $\eta Y$.

La probabilité de survie en deuxième période dépend du niveau de la pollution, sur base de la fonction de survie suivante:

$$
\pi=\pi(P)
$$

Nous supposons que $\pi(P)>0 \forall P \geq 0$. L'impact de la pollution sur les chances de survie dépend du niveau de la pollution. En dessous d'un certain seuil, $\bar{P}$, la pollution n'a pas d'impact sur les chances de survie, et $\pi=1$. Par contre, au-delà de $\bar{P}$, la pollution réduit les chances de survie:

$$
\pi(P)\left\{\begin{array}{l}
=1 \text { si } P \leq \bar{P} \\
<1 \text { si } P>\bar{P}
\end{array}\right.
$$




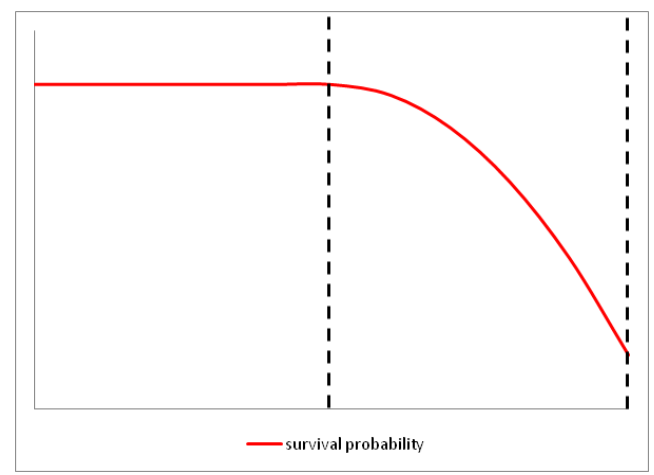

Figure 1: The survival function $\pi(P)$.

En ce qui concerne les dérivées de $\pi(P)$, nous supposons:

$$
\begin{aligned}
& \text { si } P<\bar{P}: \pi^{\prime}(P)=0 \\
& \text { si } P>\bar{P}: \pi^{\prime}(P)<0, \pi^{\prime \prime}(P)<0 \text { et } \lim _{P \rightarrow \bar{P}_{+}} \pi^{\prime}(P)=0
\end{aligned}
$$

Vu que $P=\eta A \ell$, un niveau particulier de $\ell$ est associé au seuil de pollution $\bar{P}$. Ce niveau, dénoté par $\bar{\ell}$, est tel que $\eta A \bar{\ell}=\bar{P}$. Nous avons donc $\bar{\ell}=\frac{\bar{P}}{\eta A}$. Le seuil de travail au-delà duquel la pollution associée est une cause de mortalité est croissant avec $\bar{P}$, et décroissant avec $\eta$ et $A$. Vu que $\ell$ prend 1 comme valeur maximale, la pollution maximale est égale à $P^{M A X}=\eta A$, conduisant à la plus petite probabilité de survie, égale à $\pi(\eta A) \equiv \tilde{\pi}<1$. La Figure 1 illustre une fonction de survie satisfaisant nos hypothèses. ${ }^{5}$

Les individus ont des préférences qui satisfont l'hypothèse d'utilité attendue, et qui sont additives à travers le temps. ${ }^{6}$ L'utilité de la mort étant normalisée à 0 , la fonction d'utilité prend la forme: ${ }^{7}$

$$
u(c)+\pi(P) u(d)
$$

où $c$ est la consommation de première période, $d$ est la consommation de seconde période. Nous supposons $u^{\prime}(c)>0, u^{\prime \prime}(c)<0$, ainsi que $\lim _{c \rightarrow 0} u^{\prime}(c)=+\infty$ et $\lim _{c \rightarrow+\infty} u^{\prime}(c)=0$. Nous supposons qu'il existe un seuil de consommation $\bar{c}>0$ rendant l'individu indifférent entre, d'une part, la période de vie avec $\bar{c}$ et, d'autre part, la mort (voir Becker et al 2005). $\bar{c}$ satisfait $u(\bar{c})=0$.

${ }^{5}$ Il s'agit de la fonction de survie:

$$
\pi(P)\left\{\begin{array}{l}
=1 \text { si } P \leq \bar{P} \\
=1-0.0001\left(\frac{P}{\eta A}-\frac{\bar{P}}{\eta A}\right)^{2} \text { si } P>\bar{P}
\end{array}\right.
$$

où $\frac{\bar{P}}{\eta A}=0.5$ et $\frac{P}{\eta A}=\ell$ varie entre $0,5(\bar{P})$ et $1\left(P^{M A X}\right)$.

${ }^{6} \mathrm{Vu}$ que la probabilité de survie $\pi(P)$ joue ici un rôle de taux d'escompte biologique, nous faisons abstraction des préférences temporelles pures.

${ }^{7} \mathrm{Vu}$ que le travail est déjà source de désutilité à travers la pollution, nous faisons ici abstraction de la désutilité pure du travail. 


\section{Le laissez-faire}

Nous supposons, au laissez-faire, que le travail est rémunéré à sa productivité marginale, et qu'il existe un marché d'annuités parfait, donnant un rendement actuariellement juste. Dès lors, le taux de rendement brut sur l'épargne, dénoté par $\hat{R}$, est:

$$
\hat{R}=\frac{R}{\pi(P)}
$$

où $R$ est égal à 1 plus le taux d'intérêt. Afin de simplifier l'exposition, nous supposerons ici que $R=1$.

$\mathrm{Au}$ laissez-faire, les individus choisissent une quantité de travail $\ell$ et une épargne $s$ de manière à maximiser leur bien-être attendu sur la vie, tout en satisfaisant leurs contraintes budgétaires de première et seconde périodes:

$$
\begin{aligned}
c & =A \ell-s \\
d & =\frac{s}{\pi(P)}
\end{aligned}
$$

Bien que les choix de travail affectent la taille de l'output, et, par là, le niveau de la pollution et de l'espérance de vie $1+\pi(P)$, nous allons ici supposer que les individus sont myopes, et ne voient pas l'impact de leurs décisions de production sur les conditions de survie prévalant dans l'économie. En d'autres termes, les individus prennent la probabilité de survie $\pi(P)$ comme donnée $(\pi(P)=\bar{\pi})$, alors que celle-ci dépend de leur propre mode de vie.

Le problème des individus myopes au laissez-faire s'écrit:

$$
\max _{s, \ell} u(A \ell-s)+\bar{\pi} u\left(\frac{s}{\bar{\pi}}\right)
$$

La condition de première ordre pour une épargne optimale est

$$
u^{\prime}(c)=\bar{\pi} u^{\prime}(d) \frac{1}{\bar{\pi}}=u^{\prime}(d)
$$

Il est donc ici optimal de lisser la consommation sur le cycle de vie $(c=d)$.

En ce qui concerne le choix de la quantité de travail, la condition de premier ordre est, de par la myopie des agents:

$$
u^{\prime}(c)=\frac{0}{A}
$$

Vu que $u^{\prime}(\cdot)>0$ pour tout $c \geq 0$, nous avons $u^{\prime}(d)>0$, de sorte que ces deux conditions de premier ordre ne peuvent pas être satisfaites ensemble. Comme les individus ne voient pas l'effet néfaste de la production sur leurs chances de survie, le gain marginal d'utilité associé à une quantité de travail légèrement supérieure est toujours perçu comme plus grand que la perte marginale d'utilité associée à cette hausse du travail. Les individus choisissent donc une solution de coin, qui est la quantité maximale de travail $\ell=1 .^{8} \mathrm{Il}$ s'ensuit que la probabilité de survie prend son niveau minimum: $\pi=\pi(\kappa A)=\tilde{\pi}$.

\footnotetext{
${ }^{8}$ Notons que nos résultats ne seraient pas profondément affectés par l'introduction d'une désutilité pure du travail conduisant à une solution intérieure pour $\ell$ au laissez-faire.
} 
Proposition 1 Au laissez-faire, nous avons:

$$
\begin{aligned}
c^{L F} & =d^{L F}=\frac{A}{1+\tilde{\pi}} \\
\ell^{L F} & =1>\bar{\ell} \\
P^{L F} & =\eta A>\bar{P} \\
\pi^{L F} & =\tilde{\pi}<1
\end{aligned}
$$

Proof. A partir de la condition de premier ordre pour l'épargne, nous avons $c=d$. Etant donné les contraintes budgétaires $c=A \ell-s$ et $d=\frac{s}{\pi}$, la contrainte intertemporelle $A \ell=c+\pi d$ est égale à $A \ell=c(1+\pi)$. Vu que $\ell=1$, nous avons $\pi=\tilde{\pi}$ et $c=d=\frac{A}{1+\tilde{\pi}}$.

Etant donné que $\pi^{L F}<1$, certains individus disparaissent avant d'avoir atteint l'âge de la retraite. Ces disparitions prématurées conduisent, en général, à des inégalités de bien-être réalisé sur la vie. En effet, au laissez-faire, le bienêtre réalisé d'un individu malchanceux à vie courte est égal à: $u\left(\frac{A}{1+\tilde{\pi}}\right)$, tandis que celui d'un individu chanceux à vie longue est égal à: $2 u\left(\frac{A}{1+\tilde{\pi}}\right)$. Il s'ensuit que l'individu à vie courte est pénalisé au laissez-faire lorsque:

$$
u\left(\frac{A}{1+\tilde{\pi}}\right)>0 \Longleftrightarrow \frac{A}{1+\tilde{\pi}}>\bar{c}
$$

Cette condition est très plausible dans une économie où la productivité $A$ est suffisamment élevée. Dans le reste de l'article, nous supposerons que $A>(1+$ $\tilde{\pi}) \bar{c}$, de sorte qu'au laissez-faire les individus décédés prématurément sont moins bien lotis que les individus profitant de leur retraite.

\section{L'optimum utilitariste}

Le planificateur social utilitariste choisit les niveaux d'épargne et de travail qui maximisent le bien-être social, défini comme la somme des utilités individuelles. Contrairement aux individus, le planificateur utilitariste ne souffre pas de myopie, et prend pleinement en compte l'effet de la production sur la pollution et sur les conditions de survie.

Le problème du planificateur social utilitariste s'écrit:

$$
\begin{aligned}
& \max _{c, d, \ell} u(c)+\pi(\eta A \ell) u(d) \\
& \text { s.c. } c+\pi(\eta A \ell) d=A \ell
\end{aligned}
$$

Les conditions de premier ordre pour les consommations impliquent:

$$
u^{\prime}(c)=u^{\prime}(d)
$$

Comme au laissez-faire, le lissage de la consommation sur le cycle de vie est optimal. Nous obtenons donc, en utilisant la contrainte budgétaire intertemporelle $A \ell=c+\pi d$, que $c=d=\frac{A \ell}{1+\pi(\eta A \ell)}$. 
La condition de premier ordre pour le travail est:

$$
A u^{\prime}(c)+\eta A \pi^{\prime}(\eta A \ell) u(d)+\pi(\eta A \ell) u^{\prime}(d) \frac{-s \pi^{\prime}(\eta A \ell) \eta A}{[\pi(\eta A \ell)]^{2}}=0
$$

Cette condition peut être réécrite comme:

$$
A u^{\prime}(c)=-\eta A \pi^{\prime}(\eta A \ell)\left[u(d)-u^{\prime}(d) d\right]
$$

L'étude de cette condition donne les résultats suivants.

Proposition 2 A l'optimum utilitariste,

- Si $u^{\prime}\left(\frac{A}{1+\tilde{\pi}}\right)\left[1-\frac{\eta \pi^{\prime}(\eta A) A}{1+\tilde{\pi}}\right] \geq-\eta \pi^{\prime}(\eta A)\left[u\left(\frac{A}{1+\tilde{\pi}}\right)\right]$, nous avons:

$$
\begin{aligned}
c^{U} & =d^{U}=\frac{A}{1+\tilde{\pi}} \\
\ell^{U} & =1>\bar{\ell} \\
P^{U} & =\eta A>\bar{P} \\
\pi^{U} & =\tilde{\pi}<1
\end{aligned}
$$

- Si $u^{\prime}\left(\frac{A}{1+\tilde{\pi}}\right)\left[1-\frac{\eta \pi^{\prime}(\eta A) A}{1+\tilde{\pi}}\right]<-\eta \pi^{\prime}(\eta A)\left[u\left(\frac{A}{1+\tilde{\pi}}\right)\right]$, nous avons:

$$
\begin{aligned}
c^{U} & =d^{U}=\frac{A \ell^{U}}{1+\pi\left(\eta A \ell^{U}\right)} \\
\bar{\ell} & <\ell^{U}<1 \\
P^{U} & =\eta A \ell^{U}>\bar{P} \\
\tilde{\pi} & <\pi^{U}<1
\end{aligned}
$$

où $\ell^{U}$ satisfait $\frac{u^{\prime}\left(\frac{A \ell^{U}}{1+\pi\left(\eta A \ell^{U}\right)}\right)}{u\left(\frac{A \ell^{U}}{1+\pi\left(\eta A \ell^{U}\right)}\right)}=\frac{-\eta \pi^{\prime}\left(\eta A \ell^{U}\right)}{\left[1-\frac{\eta \pi^{\prime}\left(\eta A \ell^{U}\right) A \ell^{U}}{1+\pi\left(\eta A \ell^{U}\right)}\right]}$.

Proof. En remplaçant pour $c=d=\frac{A \ell}{1+\pi(\eta A \ell)}$, la condition de premier ordre pour $\ell$ devient:

$u^{\prime}\left(\frac{A \ell}{1+\pi(\eta A \ell)}\right)=-\eta \pi^{\prime}(\eta A \ell)\left[u\left(\frac{A \ell}{1+\pi(\eta A \ell)}\right)-u^{\prime}\left(\frac{A \ell}{1+\pi(\eta A \ell)}\right) \frac{A \ell}{1+\pi(\eta A \ell)}\right]$

Cette condition peut être réécrite comme:

$$
u^{\prime}\left(\frac{A \ell}{1+\pi(\eta A \ell)}\right)\left[1-\frac{\eta \pi^{\prime}(\eta A \ell) A \ell}{1+\pi(\eta A \ell)}\right]=-\eta \pi^{\prime}(\eta A \ell)\left[u\left(\frac{A \ell}{1+\pi(\eta A \ell)}\right)\right]
$$

Lorsque $\ell$ tend vers 0 , le membre de gauche tend vers $u^{\prime}(0)\left[1-\frac{\eta 0 A 0}{2}\right]=$ $+\infty$, tandis que le membre de droite tend vers $-\eta 0[u(0)]=0$. Lorsque $\ell$ tend 
vers $\bar{\ell}$ par le bas, le membre de gauche de la condition tend vers $u^{\prime}\left(\frac{A \bar{\ell}}{2}\right)\left[1-\frac{\eta 0 A \ell}{2}\right]=$ $u^{\prime}\left(\frac{A \bar{\ell}}{2}\right)$, tandis que le membre de droite tend vers $-\eta 0\left[u\left(\frac{A \bar{\ell}}{2}\right)\right]=0$. En fait, comme $\lim _{P \rightarrow \bar{P}-} \pi^{\prime}(P)=0$, le membre de gauche $u^{\prime}\left(\frac{A \ell}{1+\pi(\eta A \ell)}\right)$ est toujours supérieur au membre de droite, de sorte que le $\ell$ optimal n'est pas compris dans l'intervale $[0, \bar{\ell}[$.

Lorsque $\ell$ tend vers $\bar{\ell}$ par le haut, le membre de gauche de la condition tend vers $u^{\prime}\left(\frac{A \bar{\ell}}{2}\right)\left[1-\frac{\eta 0 A \ell}{2}\right]=u^{\prime}\left(\frac{A \bar{\ell}}{2}\right)$, tandis que le membre de droite tend vers $-\eta 0\left[u\left(\frac{A \bar{\ell}}{2}\right)\right]=0$. Le membre de gauche est supérieur au membre de droite, car $u^{\prime}\left(\frac{A \bar{\ell}}{2}\right)>0$. Il est donc socialement optimal d'avoir $\ell>\bar{\ell}$. Lorsque $\ell$ tend vers 1, le membre de gauche de la condition tend vers $u^{\prime}\left(\frac{A}{1+\tilde{\pi}}\right)\left[1-\frac{\eta \pi^{\prime}(\eta A) A}{1+\tilde{\pi}}\right]$, tandis que le membre de droite tend vers $-\eta \pi^{\prime}(\eta A)\left[u\left(\frac{A}{1+\tilde{\pi}}\right)\right]$. Dès lors, plusieurs cas peuvent se produire:

- Si $u^{\prime}\left(\frac{A}{1+\tilde{\pi}}\right)\left[1-\frac{\eta \pi^{\prime}(\eta A) A}{1+\tilde{\pi}}\right] \geq-\eta \pi^{\prime}(\eta A)\left[u\left(\frac{A}{1+\tilde{\pi}}\right)\right]$, le $\ell$ optimal est égal à 1 ;

- Si $u^{\prime}\left(\frac{A}{1+\tilde{\pi}}\right)\left[1-\frac{\eta \pi^{\prime}(\eta A) A}{1+\tilde{\pi}}\right]<-\eta \pi^{\prime}(\eta A)\left[u\left(\frac{A}{1+\tilde{\pi}}\right)\right]$, le $\ell$ optimal appartient à l'intervale $] \bar{\ell}, 1[$. En effet, le membre de gauche excède le membre de droite pour $\ell$ proche de $\bar{\ell}$, mais est inférieur au membre de droite pour $\ell=1$, de sorte que, par continuité, il existe un $\ell$ optimal intérieur satisfaisant $u^{\prime}\left(\frac{A \ell}{1+\pi(\eta A \ell)}\right)\left[1-\frac{\eta \pi^{\prime}(\eta A \ell) A \ell}{1+\pi(\eta A \ell)}\right]=-\eta \pi^{\prime}(\eta A \ell)\left[u\left(\frac{A \ell}{1+\pi(\eta A \ell)}\right)\right]$.

Une fois $\ell$ connu, il est possible de dériver $c=d=\frac{A \ell}{1+\pi(\eta A \ell)}$.

Si $u^{\prime}\left(\frac{A}{1+\tilde{\pi}}\right)\left[1-\frac{\eta \pi^{\prime}(\eta A) A}{1+\tilde{\pi}}\right] \geq-\eta \pi^{\prime}(\eta A)\left[u\left(\frac{A}{1+\tilde{\pi}}\right)\right]$, on a $c=d=\frac{A}{1+\tilde{\pi}}$.

Si $u^{\prime}\left(\frac{A}{1+\tilde{\pi}}\right)\left[1-\frac{\eta \pi^{\prime}(\eta A) A}{1+\tilde{\pi}}\right]<-\eta \pi^{\prime}(\eta A)\left[u\left(\frac{A}{1+\tilde{\pi}}\right)\right]$, on a $c=d=\frac{A \ell}{1+\pi(\eta A \ell)}$ avec $\ell$ satisfaisant $u^{\prime}\left(\frac{A \ell}{1+\pi(\eta A \ell)}\right)\left[1-\frac{\eta \pi^{\prime}(\eta A \ell) A \ell}{1+\pi(\eta A \ell)}\right]=-\eta \pi^{\prime}(\eta A \ell)\left[u\left(\frac{A \ell}{1+\pi(\eta A \ell)}\right)\right]$.

La condition peut être écrite comme $\frac{u^{\prime}(d)}{u(d)} \gtrless \frac{-\eta \pi^{\prime}(P)}{\left[1-\eta \pi^{\prime}(P) d\right]}$, de sorte que la structure de l'optimum utilitariste dépend de la forme précise des fonctions $u(\cdot)$ et $\pi(\cdot)$. Notons également que, dans les économies pauvres, la productivité $A$ est faible, de sorte que $u^{\prime}\left(\frac{A}{1+\tilde{\pi}}\right)$ est très élevé et $\left|\pi^{\prime}(\eta A)\right|$ est faible. Il s'ensuit que le premier cas - où l'optimum utilitariste coïncide avec le laissez-faire - est ici davantage probable. Par contre, dans les économies avancées, où $A$ est élevé, $u^{\prime}\left(\frac{A}{1+\tilde{\pi}}\right)$ est davantage faible et $\left|\pi^{\prime}(\eta A)\right|$ est plus élevé, de sorte que l'optimum utilitariste est davantage susceptible d'appartenir au second cas.

Sur base des Propositions 1 et 2, la comparaison avec le laissez-faire est immédiate. Comme le montre la Proposition 3, l'optimum utilitariste coïncide avec le laissez-faire lorsque $u^{\prime}\left(\frac{A}{1+\tilde{\pi}}\right)\left[1-\frac{\eta \pi^{\prime}(\eta A) A}{1+\tilde{\pi}}\right] \geq-\eta \pi^{\prime}(\eta A)\left[u\left(\frac{A}{1+\tilde{\pi}}\right)\right]$; par 
contre, il est associé à des consommations plus petites, à une production et une pollution moindre, et à une espérance de vie plus élevée qu'au laissez-faire lorsque $u^{\prime}\left(\frac{A}{1+\tilde{\pi}}\right)\left[1-\frac{\eta \pi^{\prime}(\eta A) A}{1+\tilde{\pi}}\right]<-\eta \pi^{\prime}(\eta A)\left[u\left(\frac{A}{1+\tilde{\pi}}\right)\right]$.

Proposition 3 Comparons le laissez-faire et l'optimum utilitariste.

- Si $u^{\prime}\left(\frac{A}{1+\tilde{\pi}}\right)\left[1-\frac{\eta \pi^{\prime}(\eta A) A}{1+\tilde{\pi}}\right] \geq-\eta \pi^{\prime}(\eta A)\left[u\left(\frac{A}{1+\tilde{\pi}}\right)\right]$, nous avons:

$$
\begin{aligned}
c^{U} & =d^{U}=c^{L F}=d^{L F}=\frac{A}{1+\tilde{\pi}} \\
\ell^{U} & =\ell^{L F}=1>\bar{\ell} \\
P^{U} & =P^{L F}=\eta A>\bar{P} \\
\pi^{U} & =\pi^{L F}=\tilde{\pi}<1
\end{aligned}
$$

- Si $u^{\prime}\left(\frac{A}{1+\tilde{\pi}}\right)\left[1-\frac{\eta \pi^{\prime}(\eta A) A}{1+\tilde{\pi}}\right]<-\eta \pi^{\prime}(\eta A)\left[u\left(\frac{A}{1+\tilde{\pi}}\right)\right]$, nous avons:

$$
\begin{aligned}
c^{U} & =d^{U}=\frac{A \ell^{U}}{1+\pi\left(\eta A \ell^{U}\right)}<c^{L F}=d^{L F}=\frac{A}{1+\tilde{\pi}} \\
\bar{\ell} & <\ell^{U}<\ell^{L F}=1 \\
\bar{P} & <P^{U}=\eta A \ell^{U}<P^{L F}=\eta A \\
\tilde{\pi} & =\pi^{L F}<\pi^{U}<1
\end{aligned}
$$

Proof. La preuve repose sur la comparaison des Propositions 1 et 2.

Notons cependant que, même si l'optimum utilitariste est, sous certaines conditions, associé à une mortalité moindre qu'au laissez-faire, il n'en demeure pas moins que la pollution conduit, même à l'optimum utilitariste, à des décès prématurés. Il en résulte un écart de bien-être réalisé entre les individus chanceux à vie longue et les individus malchanceux à vie courte.

Lorsque $u^{\prime}\left(\frac{A}{1+\tilde{\pi}}\right)\left[1-\frac{\eta \pi^{\prime}(\eta A) A}{1+\tilde{\pi}}\right] \geq-\eta \pi^{\prime}(\eta A)\left[u\left(\frac{A}{1+\tilde{\pi}}\right)\right]$, cet écart est positif si et seulement si:

$$
u\left(\frac{A}{1+\tilde{\pi}}\right)>0
$$

Cette condition est identique à celle prévalant au laissez-faire.

Lorsque $u^{\prime}\left(\frac{A}{1+\tilde{\pi}}\right)\left[1-\frac{\eta \pi^{\prime}(\eta A) A}{1+\tilde{\pi}}\right]<-\eta \pi^{\prime}(\eta A)\left[u\left(\frac{A}{1+\tilde{\pi}}\right)\right]$, cet écart est positif si et seulement si:

$$
u\left(\frac{A \ell^{U}}{1+\pi\left(\eta A \ell^{U}\right)}\right)>0
$$

Les inégalités de bien-être réalisé sont ici réduites par rapport au laissez-faire, car la consommation en seconde période est plus faible qu'au laissez-faire, de par une production moindre et une plus grande proportion de retraités. 


\section{$5 \quad$ L'optimum égalitarien ex post}

Au laissez-faire comme à l'optimum utilitariste, certains individus décèdent avant d'avoir atteint la seconde période de vie. Ces décès précoces sont causés par un niveau de pollution excédant le seuil $\bar{P}$. Notons que si tous les jeunes adultes contribuent ici également au processus de pollution, seuls certains d'entre eux sont victimes de la pollution ainsi générée. Il en résulte des inégalités de bien-être réalisé sur la vie plus ou moins importantes.

Ces inégalités prévalant ex post sont inacceptables: comment justifier que, parmi des individus égaux ex ante, certains d'entre eux - ceux qui ont une vie courte - sont fortement désavantagés par rapport aux autres? La responsabilité de chacun est engagée - chacun contribue au processus de pollution, y compris ceux qui décèdent prématurément - mais l'inégalité qui en résulte en termes de bien-être ex post n'est pas juste. Face à une situation où des individus égaux ex ante se retrouvent $e x$ post avec des niveaux de bien-être réalisés très différents, un planificateur social peut estimer que ces écarts de bien-être relèvent de l'arbitraire de la nature, et va chercher à minimiser ceux-ci.

Le problème du planificateur égalitarien ex post consiste à choisir les niveaux de production et de consommation de manière à maximiser le niveau de bienêtre sur la vie des personnes les moins bien loties. Si on considère une économie où $\pi<1$, le problème est simple, car les individus les moins bien lotis sont généralement ceux qui bénéficient d'une vie courte. Le planificateur peut donc maximiser leur bien-être en transférant les ressources vers les âges jeunes, de manière à compenser ceux qui disparaissent plus tôt (voir Fleurbaey et al 2014a).

Cependant, dans le présent contexte, la proportion de survivants dépend du niveau de pollution, qui est choisi par le planificateur. Le niveau de bien-être minimum dans l'économie considérée prend donc la forme suivante:

$$
\min U=\left\{\begin{array}{l}
u(c)+u(d) \text { si } P \leq \bar{P} \text { ou } \ell \leq \bar{\ell} \\
\min \{u(c), u(c)+u(d)\} \text { si } P>\bar{P} \text { ou } \ell>\bar{\ell}
\end{array}\right.
$$

Il existe donc, dans le présent contexte, deux manières distinctes de maximiser le bien-être des individus les moins bien lotis. Une première manière consiste à produire de manière à ce que la pollution soit inférieure ou égale au seuil $\bar{P}$, ce qui revient à travailler une quantité $\ell \leq \bar{\ell}$. Dans ce cas, tous les individus bénéficient d'une vie longue $(\pi=1)$. Une seconde manière consiste à produire bien plus $(\ell>\bar{\ell})$, générant ainsi une pollution supérieure au seuil $\bar{P}$, et à transférer une grande partie des ressources produites vers les âges jeunes, de façon à compenser ceux qui auront, du fait de cette pollution, une vie plus courte. La question est donc de savoir dans quel cas $-P \leq \bar{P}$ ou $P>\bar{P}$ - les personnes les moins bien loties ont le niveau de bien-être réalisé le plus élevé.

Considérons le premier cas, où $\ell \leq \bar{\ell}$. Dans ce cas, tous les membres de la population bénéficient de la même durée de vie. Le niveau de bien-être de tous 
les individus est égal à $u(c)+u(d)$. Dès lors, le problème du planificateur est:

$$
\begin{aligned}
& \max _{\ell, c, d} u(c)+u(d) \\
& \text { s.c. } 0 \leq \ell \leq \bar{\ell} \\
& \text { s.c. } A \ell=c+d
\end{aligned}
$$

Vu que le Lagrangien associé est croissant en $\ell$ pour des valeurs de $\ell \in[0, \bar{\ell}]$, la solution de ce problème est donnée par:

$$
\begin{aligned}
\ell^{*} & =\bar{\ell} \\
c^{*} & =d^{*}=\frac{A \bar{\ell}}{2} \\
\pi^{*} & =1
\end{aligned}
$$

Il s'ensuit que le bien-être maximum des individus les moins bien lotis (ici toute la population) est ici égal à:

$$
2 u\left(\frac{A \bar{\ell}}{2}\right)
$$

Considérons le second cas, où $\ell>\bar{\ell}$. Dans ce cas, la population se divise $e x$ post en deux groupes. D'une part, des individus qui vivent une seule période, et dont le bien-être est $u(c)$. D'autre part, des individus qui vivent deux périodes, et dont le bien être est $u(c)+u(d)$. Le problème du planificateur est donc:

$$
\begin{aligned}
& \max _{\ell, c, d} \min \{u(c), u(c)+u(d)\} \\
& \text { s.c. } \bar{\ell}<\ell \leq 1 \\
& \text { s.c. } A \ell=c+\pi(\eta A \ell) d
\end{aligned}
$$

La fonction objectif est ici non différentiable. Mais ce problème peut être réécrit sous une forme plus appropriée. Pour ce faire, notons que la condition $u(d)=0$ est nécessaire et suffisante pour égaliser le niveau de bien-être réalisé des membres des deux groupes. Cette condition équivaut à $d=\bar{c}$. Dès lors, sous l'hypothèse que l'économie est suffisamment productive $(A>(1+\tilde{\pi}) \bar{c})$, le problème peut être réécrit comme un problème de maximisation de la consommation de première période $c$ sous la contrainte de ressources, sous la contrainte $\ell>\bar{\ell}$ et sous la contrainte égalitarienne $d=\bar{c}$, qui assure que les individus à vie longue ne sont ni mieux ni moins bien lotis que les individus à vie courte. Etant donné que $c=A \ell-(A \ell \eta) d$ et que $d=\bar{c}$, le problème peut s'écrire comme:

$$
\begin{aligned}
& \max _{\ell} A \ell-\pi(\eta A \ell) \bar{c} \\
& \text { s.c. } \bar{\ell}<\ell \leq 1
\end{aligned}
$$

Vu que, pour $\ell>\bar{\ell}$, nous avons $\pi^{\prime}(\eta A \ell)<0$, il s'ensuit que la fonction objectif est croissante en $\ell$ pour des valeurs de $\ell \in[\bar{\ell}, 1]$. Par conséquent, l'optimum 
égalitarien ex post est le suivant:

$$
\begin{aligned}
\ell^{* *} & =1 \\
c^{* *} & =A-\tilde{\pi} \bar{c} \\
d^{* *} & =\bar{c} \\
\pi^{* *} & =\tilde{\pi}<1
\end{aligned}
$$

Cet optimum inclut, comme au laissez-faire, $\ell=1$, et conduit à la même mortalité qu'au laissez faire $(\pi=\tilde{\pi})$. Mais il est très différent du laissez-faire, au sens où le profil de consommation est ici décroissant avec l'âge, et non plus plat comme au laissez-faire. Dans ce cas, le bien-être des moins bien lotis est égal à:

$$
u(A-\tilde{\pi} \bar{c})
$$

Au final, l'option la plus favorable pour le planificateur égalitarien ex post dépend de savoir si:

$$
2 u\left(\frac{A \bar{\ell}}{2}\right) \gtrless u(A-\tilde{\pi} \bar{c})
$$

Le choix est donc entre, d'une part, produire au seuil critique et répartir ces quelques ressources également à travers les âges, et, d'autre part, produire au dessus du seuil critique et concentrer ces ressources aux jeunes âges, afin de neutraliser l'effet des décès prématurés. La Proposition 4 identifie les conditions sous lesquelles les deux options sont les plus souhaitables.

Proposition 4 Considérons l'optimum égalitarien ex post. Définissions $\hat{\ell}=$ $\frac{2 u^{-1}\left(\frac{u(A-\tilde{\pi} \bar{c})}{2}\right)}{A}$.

- Si $\bar{\ell}>\hat{\ell}$, alors

$$
\begin{aligned}
\ell^{P O S T} & =\ell^{*}=\bar{\ell} \\
c^{P O S T} & =d^{P O S T}=c^{*}=d^{*}=\frac{A \bar{\ell}}{2} \\
\pi^{P O S T} & =\pi^{*}=1
\end{aligned}
$$

- Si $\bar{\ell}<\hat{\ell}$, alors

$$
\begin{aligned}
\ell^{P O S T} & =\ell^{* *}=1 \\
c^{P O S T} & =c^{* *}=A-\tilde{\pi} \bar{c} \\
d^{P O S T} & =d^{* *}=\bar{c} \\
\pi^{\text {POST }} & =\pi^{* *}=\tilde{\pi}<1
\end{aligned}
$$

- Si $\bar{\ell}=\hat{\ell}$, fixer $\ell^{P O S T}=\bar{\ell}$ ou $\ell^{P O S T}=1$ génère le même bien-être pour les moins bien lotis. 
Proof. Les deux alternatives génèrent le même niveau de bien-être pour le moins bien loti lorsque: $2 u\left(\frac{A \bar{\ell}}{2}\right)=u(A-\tilde{\pi} \bar{c})$. Isolant $\bar{\ell}$, nous obtenons: $\bar{\ell}=$ $\frac{2 u^{-1}\left(\frac{u(A-\tilde{\pi} \bar{c})}{2}\right)}{A}=\hat{\ell}$. Lorsque $\bar{\ell}>\frac{2 u^{-1}\left(\frac{u(A-\tilde{\pi} \bar{c})}{2}\right)}{A}$, la première alternative donne un niveau de bien-être réalisé pour le moins bien loti supérieur. Lorsque $\bar{\ell}<$ $\frac{2 u^{-1}\left(\frac{u(A-\tilde{\pi} \bar{c})}{2}\right)}{A}$, c'est la deuxième option qui est préférable.

La forme de l'optimum égalitarien ex post dépend du seuil $\bar{\ell}$ en-dessous duquel la pollution ne conduit pas à des décès prématurés. Si ce seuil est élevé $(\bar{\ell}>\hat{\ell})$, il est optimal, d'un point de vue ex post égalitarien, de faire travailler les individus au niveau de ce seuil $\bar{\ell}$, de manière à maximiser le bien-être sur la vie des individus, qui, sous cette option, profitent tous de la même durée de vie. Par contre, si le seuil $\bar{\ell}$ est faible $(\bar{\ell}<\hat{\ell})$, alors l'optimum égalitarien ex post requiert une quantité de travail supérieure à $\bar{\ell}$, conduisant à des décès prématurés. En effet, dans ce cas, la survie de tous - impliquant $\ell \leq \bar{\ell}$ - se ferait au détriment des moins bien lotis, à cause de consommations trop faibles. Ceci explique qu'il est alors optimal, dans l'intérêt des moins bien lotis, d'augmenter la production et la pollution au delà du seuil $\bar{P}$.

Notons que le seuil $\hat{\ell}$ est décroissant avec $\tilde{\pi}$. Plus la proportion de survivants au cas où la pollution est maximale est élevée, et plus le seuil $\hat{\ell}$ est faible, ce qui rend la première option - la pollution maintenue à $\bar{P}$ - davantage souhaitable. ${ }^{9}$ Le seuil $\hat{\ell}$ est décroissant en $\bar{c}$. Si les individus assignent une grande valeur à la survie, $\bar{c}$ est faible, ce qui augmente le seuil $\hat{\ell}$, rendant la première option - la pollution maintenue à $\bar{P}$ - moins attractive. L'intuition derrière ce résultat surprenant est la suivante. Un plus faible $\bar{c}$ rend l'option "pollution au delà de $\bar{P}$ " plus attractive pour les moins bien lotis, car un faible $\bar{c}$ permet au planificateur égalitarien de redistribuer davantage les ressources vers les jeunes.

La Proposition 5 compare l'optimum égalitarien ex post avec le laissez-faire.

Proposition 5 Comparons l'optimum égalitarien ex post avec le laissez-faire:

- $S i \bar{\ell}>\hat{\ell}$, alors

$$
\begin{aligned}
\ell^{P O S T} & =\bar{\ell}<\ell^{L F}=1 \\
c^{P O S T} & <c^{L F} ; d^{P O S T}<d^{L F} \\
\pi^{P O S T} & =1>\pi^{L F}
\end{aligned}
$$

- $S i \bar{\ell}<\hat{\ell}$, alors

$$
\begin{aligned}
\ell^{P O S T} & =\ell^{L F}=1 \\
c^{P O S T} & >c^{L F} ; d^{P O S T}<d^{L F} \\
\pi^{P O S T} & =1=\pi^{L F}
\end{aligned}
$$

Proof. La première partie de la proposition découle de $c^{P O S T}=\frac{A \bar{\ell}}{2}$ et $c^{L F}=$ $\frac{A}{1+\tilde{\pi}}$. Vu que $\bar{\ell}<1$ et $\tilde{\pi}<1$, on a $c^{P O S T}<c^{L F}$. Le même raisonnement

${ }^{9}$ L'intuition est que si $\tilde{\pi}$ est élevé, polluer au delà de $\bar{P}$ - et donc réduire la durée de vie des moins bien lotis - ne permet pas d'améliorer le sort de ceux-ci. 
prévaut pour $d^{P O S T} \lessgtr d^{L F}$. En ce qui concerne la seconde partie, l'hypothèse $\bar{c}(1+\tilde{\pi})<A$ implique $c^{P O S T}>c^{L F}$ et $d^{P O S T}<d^{L F}$.

Si le seuil $\bar{\ell}$ au dessus duquel la pollution devient une cause de décès prématuré est élevé, l'optimum égalitarien ex post implique une quantité de travail plus faible qu'au laissez-faire, des consommations plus faibles, mais une espérance de vie plus grande. Par contre, si le seuil $\bar{\ell}$ est faible, le laissez-faire et l'optimum égalitarien ex post impliquent les mêmes niveaux de production, de pollution et d'espérance de vie. Par contre, les profils de consommation sont différents: la consommation aux jeunes âges est plus élevée qu'au laissez-faire sous l'optimum ex post, mais plus faible aux âges élevés.

Proposition 6 Comparons l'optimum égalitarien ex post avec l'optimum utilitariste sous $u^{\prime}\left(\frac{A}{1+\tilde{\pi}}\right)\left[1-\frac{\eta \pi^{\prime}(\eta A) A}{1+\tilde{\pi}}\right]<-\eta \pi^{\prime}(\eta A)\left[u\left(\frac{A}{1+\tilde{\pi}}\right)\right]$ :

- Si $\bar{\ell}>\hat{\ell}$, alors

$$
\begin{aligned}
\ell^{P O S T} & =\bar{\ell}<\ell^{U} \\
c^{P O S T} & <c^{U} ; d^{P O S T}<d^{U} \\
\pi^{P O S T} & =1>\pi^{U}
\end{aligned}
$$

- Si $\bar{\ell}<\hat{\ell}$, alors

$$
\begin{aligned}
\ell^{P O S T} & =1>\ell^{U} \\
c^{P O S T} & \lessgtr c^{U} ; d^{P O S T} \lessgtr d^{U} \\
\pi^{P O S T} & =\tilde{\pi}<\pi^{U}
\end{aligned}
$$

Proof. Dans la première partie de la proposition, $c^{P O S T}<c^{U}$ provient du fait que $\frac{A \bar{\ell}}{2}<\frac{A \ell^{U}}{1+\pi\left(\eta A \ell^{U}\right)}$ pour $\bar{\ell}<\ell^{U}$. De même, l'inégalité $d^{P O S T}<d^{U}$ provient de $\frac{A \bar{\ell}}{2}<\frac{A \ell^{U}}{1+\pi\left(\eta A \ell^{U}\right)}$ pour $\bar{\ell}<\ell^{U}$.

Si le seuil $\bar{\ell}$ au-delà duquel la pollution devient une cause de décès est supérieur à $\hat{\ell}$, l'optimum égalitarien ex post implique moins de production et de pollution que sous l'optimum utilitariste, une espérance de vie plus élevée, mais au prix de consommations plus petites. Par contre, si $\bar{\ell}$ est inférieur à $\hat{\ell}$, l'optimum égalitarien ex post implique davantage de production et de pollution, ainsi qu'une espérance de vie plus petite.

\section{Conclusions}

La pollution constitue une cause de surmortalité de premier ordre. La présente étude a abordé la question de la compensation des individus victimes d'une surmortalité causée par la pollution. Pour ce faire, nous avons caractérisé, dans un simple modèle du cycle de vie avec une pollution affectant les chances de survie, l'optimum égalitarien ex post, et nous l'avons comparé au laissez-faire, ainsi qu'à l'optimum utilitariste. 
Notre analyse a révélé que l'optimum égalitarien ex post peut comporter une pollution soit inférieure soit égale à celle prévalant au laissez-faire. De plus, l'optimum égalitarien ex post peut être caractérisé par une pollution et une mortalité soit plus faibles, soit plus élevées qu'à l'optimum utilitariste. La comparaison des deux optima sociaux requiert donc une analyse précise des caractéristiques du processus de pollution étudié. Ces caractéristiques incluent non seulement le niveau critique $\bar{\ell}$, en dessous duquel la pollution a un effet nul sur la mortalité, mais également les caractéristiques $\tilde{\pi}$, et $\bar{c}$, qui détermine le seuil $\hat{\ell}$, et, par là, la forme précise de l'optimum égalitarien ex post.

\section{References}

Becker, G., Philipson, T, \& Soares, R. (2005). The quantity and the quality of life and the evolution of world inequality. American Economic Review, 95: 277-291.

Costa, D. \& Steckel, R. (1997). Long-term trends in health, welfare and economic growth in the United States. In: R. Steckel \& R. Floud (Eds.): Health and Welfare during the Industrialization, University of Chicago Press.

Finkelstein, M., Jerrett, M., DeLuca, P., Finkelstein, N., Verma, D., Chapman, K. \& Sears, M. (2003). Relation between income, air pollution an mortality: a cohort study. Canadian Medical Association Journal, 169: 397-402.

Fleurbaey, M. (2008). Fairness, Responsibility and Welfare. Oxford University Press, New-York.

Fleurbaey, M. \& Gaulier, G. (2009). Intertemporal comparisons of living standards by equivalent incomes. Scandinavian Journal of Economics, 111, 597-624.

Fleurbaey, M., Leroux, M.L., \& Ponthiere, G. (2014). Compensating the dead. Journal of Mathematical Economics, 51: 28-41.

Fleurbaey, M. Leroux, M.L., Pestieau, P. \& Ponthiere, G. (2014). Fair retirement under risky lifetime. International Economic Review, forthcoming.

Fleurbaey, M. \& Ponthiere, G. (2013). Prevention against equality? Journal of Public Economics, 103: 68-84.

Laden, F., Schwartz, J., Spelzer, F. \& Dockery, D. (2006). Reductions in fine particulate air pollution and mortality. Extended follow-up of the Harvard six cities study. American Journal of Respiratory and Critical Care Medicine, 173: 667-672.

Organisation Mondiale de la Santé (2014). 7 million deaths annually linked to air pollution, disponible sur http://www.who.int/phe/health_topics/outdoorair/databases/en/

Sartor, F. (2002). Les déterminants environnementaux de la mortalité, dans Caselli, G., Vallin, J., Wunsch, G. (eds.) Démographie: Analyse et Synthèse. Volume III: Les déterminants de la mortalité, Editions de l'INED, Paris.

Sen, A.K. (1998). Mortality as an indicator of economic success and failure. Economic Journal, 108: 1-25.

Usher, D. (1973). An imputation to the measure of economic growth for changes in life expectancy. In M. Moss (Ed.): The Measurement of Economic and Social Performance, NBER Studies in Income and Wealth, vol. 38.

Usher, D. (1980). The Measurement of Economic Growth. Columbia University Press, New-York. 